\title{
CONVENTIONAL EXTRACTION OF BETALAIN COMPOUNDS FROM BEETROOT PEELS WITH AQUEOUS ETHANOL SOLVENT
}

\author{
M. M. ZIN*, E. MÁRKI and Sz. BÁNVÖLGYI \\ Department of Food Engineering, Faculty of Food Science, Szent István University, Ménesi Street 44, \\ H-1118 Budapest. Hungary
}

(Received: 23 August 2019; accepted: 27 January 2020)

\begin{abstract}
Consumer concern over artificial food additives has stimulated production of pigments from natural sources. The aim of this study was to examine the effects of process variables on the content of colour compounds (betaxanthin and betacyanin) in beetroot peel juice extracted by conventional method. In this research, the extraction processes were carried out according to the central composite design with different process variables. Quantitative measurements of the basic colour compounds in beetroot extract were performed using spectrophotometer. From our experiment, it was found that the most adequate extraction conditions, which gave the highest yield of colour compounds (952.5 $\mathrm{mg} \mathrm{l}^{-1}$ of betaxanthin and $1361 \mathrm{mg} \mathrm{l}^{-1}$ of betacyanin), were extraction time $1 \mathrm{~h}$, operating temperature $20^{\circ} \mathrm{C}$, and solvent ratio $0.8 \mathrm{w} / \mathrm{v}$. Being a conventional heating method, it is a simple and cost efficient process with relatively high yield.
\end{abstract}

Keywords: beetroot, conventional extraction, betaxanthin, betacyanin

Food safety remains a significant issue especially in developing countries, since the application of food additives continues to increase. However, it is impossible to avoid the application of food colours in food industry, since colour is an important sensory attribute. Natural colorants came into consideration as soon as consumers showed more interest in organic pigments. Nevertheless, their high level of sensitivity to heat, $\mathrm{pH}$, and light, high cost, and low stability are drawbacks for their use.

Common plant pigments, such as betalains, carotenoids, anthocyanins, and carminic acids, are commonly used as natural colourants in medicinal and food applications (STINTZING \& CARLE, 2004). Among them, betalain, a water soluble compound, is prominent for its biological properties such as antioxidant, anticancer, antiradical, and antimicrobial activities (GEOrgIEV et al., 2010). This kind of compound is present in 37 species of 8 genera in the Amaranthaceae family, which is one of the 17 families of the order Caryophyllales (to date) (SLImen et al., 2017; CAI et al., 2005b) and is noticeably found in the beetroot crops, which represent an important source of bioavailable compounds. Nowadays, red beetroots are receiving significant attention from consumers due to their prominent antioxidant activity and high oxygen radical absorbance capacity (GEORGIEV et al., 2010).

Betalain is composed of a nitrogenous core structure, betalamic acid, which combines with amino acid and derives red violet betacyanin (betanin 75-95\%) and yellow betaxanthin (vulgaxanthin I $\sim 95 \%$ ) (CAI et al., 2005a). Owing to their hydrophilic nature, betalain compounds can be extracted with pure water, aqueous methanol, or ethanol. However, the extraction method and choice of solvents affect noticeably the content of individual

\footnotetext{
* To whom correspondence should be addressed

Phone: +36 70 2848794; e-mail: zinlay.net.mm@gmail.com
} 
compounds in the extract. In addition, extracted beetroots are susceptible to $\mathrm{pH}$, water activity, temperature, light, oxygen, metal ions, and enzymatic activities (RAVICHANDRAN et al., 2013).

Extraction of valuable compounds has recently been realised by ultrasonic extraction method (SIVAKUMAR et al., 2009), microwave assisted extraction method (SINGH et al., 2017), and pulse electric field extraction method (LóPEz et al., 2009). Undoubtedly, these methods have high efficiency; however, some of them are rather costly compared to traditional extraction methods.

Many observations have been made for the extraction of betalain compounds and phytochemicals from the whole tuber of beetroot. Moreover, there are some accessible investigations of bioactive antioxidants and colour-giving compounds, which are present in the waste part of beetroot such as peel (SINGH et al., 2017), pomace (KusHWAHA et al., 2018), and stalk (MARAN \& PRIYA, 2016). Nevertheless, there is still need to do further investigations of important colour compounds extraction from waste parts of beetroots. The aim of this study was to determine the effects of process variables (solvent ratio, temperature, and extraction time) on the quantities of betalain compounds in beetroot peel extracts.

\section{Materials and methods}

\subsection{Sample acquisition and preparation}

Beetroots (Beta vulgaris L., Rhonda type, harvested in 2019) were collected from local supermarket and transported to the laboratory for subsequent analysis. The purchased raw beetroots were cleaned gently with sponge to remove dust and debris. The cleaned beetroots were peeled with a sharp knife about $1 \mathrm{~mm}$ thick manually. The peels were then blended in a mixer for 2 min to obtain a pulp.

\subsection{Analytical procedures}

The pulp (50 g) was mixed with (15\%) aqueous ethanol in different peel-to-solvent ratios $(0.2,0.6,1.0 \mathrm{w} / \mathrm{v})$. The extraction processes were carried out with a thermostat water bath maintaining temperature at $20^{\circ} \mathrm{C}, 35^{\circ} \mathrm{C}, 50^{\circ} \mathrm{C}$ and three different operation times $(1 \mathrm{~h}, 3 \mathrm{~h}, 5$ h) with stirring at 215 r.p.m. After centrifuging the extracted juices for 25 minutes at 6000 r.p.m., the coloured supernatant was collected and kept under refrigeration prior to instrumental analysis.

\subsection{Spectrophotometry analysis}

Spectrophotometric analysis for quantification of two basic colour compounds was done by using DR/2400 UV-visible spectrophotometer. The absorbance values were recorded at 480 $\mathrm{nm}$ for betaxanthin compound (KUSHWAHA et al., 2018) and $535 \mathrm{~nm}$ for betacyanin compound (SINGH et al., 2017). Appropriate dilutions were done for each type of measurements with distilled water. And the concentrations of the respective betalain compounds were calculated according to the following equation as determined by RAVICHANDRAN and co-workers (2013):

$$
\mathrm{BC}=\frac{\mathrm{A} \times \mathrm{MW} \times \mathrm{DF} \times 1000}{\varepsilon \times \mathrm{L}}
$$


where $A$ is the absorbance; $M W$ is the molecular weight; $D F$ is the dilution factor; $\varepsilon$ is the molar extinction coefficient, and $L$ is path length. For quantification of betaxanthin and betacyanin, molar extinction coefficients in water and molecular weights were applied as $\varepsilon=48000 \mathrm{lmol}^{-1} \mathrm{~cm}^{-1}$ and $M W=308 \mathrm{~g} \mathrm{~mol}^{-1}, \varepsilon=600001 \mathrm{~mol}^{-1} \mathrm{~cm}^{-1}$, and $M W=550 \mathrm{~g} \mathrm{~mol}^{-1}$, respectively.

\subsection{Statistical evaluations}

Empirical design of experiment: Response Surface Methodology was used as a statistical tool to optimize the multivariable process. RSM is one of the statistical tools that are mainly used for the modelling and investigation of multivariable systems in which a response of interest is influenced by several variables (SINGH et al., 2017).

\section{Results and discussion}

\subsection{Spectrophotometric analysis}

The significance test of model fit was performed by central composite design of Design Expert software version 11.0.3, based on analysis of variance (ANOVA). The cubic model gave false results, whereas the quadratic model was statistically highly significant and so was applied for the next analysis. Based on the small P-value for the quadratic terms, we decided to fit the second-order model to the yield response. It can be deduced that the models are significant as the F value is high and the Prob $>F$ value is less than 0.0001 (Table 1). Lack of fit is non-significant, meaning that the model is significant for three variables, whereas the high coefficient of determination $\left(\mathrm{R}^{2}\right)$ account for 0.8758 and 0.9181 for the total variation in betaxanthin and betacyanin extraction, respectively.

Table 1. ANOVA values and regression coefficients of second-order polynomial model in terms of coded form for the response variables

\begin{tabular}{lccccccc}
\hline Variable & DF & \multicolumn{2}{c}{ Estimated coefficient } & \multicolumn{2}{c}{ F value } & \multicolumn{2}{c}{ P value } \\
& & Betaxanthin & Betacyanin & Betaxanthin & Betacyanin & Betaxanthin & Betacyanin \\
\hline Model & 4 & 25.87 & 34.43 & 26.45 & 42.02 & $<0.0001$ & $<0.0001$ \\
A & 1 & -0.88 & -1.45 & 1.35 & 3.7 & 0.2642 & 0.0737 \\
B & 1 & -0.90 & -1.56 & 1.42 & 4.3 & 0.2516 & 0.0558 \\
C & 1 & 5.99 & 7.19 & 62.66 & 90.89 & $<0.0001$ & $<0.0001$ \\
C $^{2}$ & 1 & -6.80 & -8.87 & 40.35 & 69.19 & $<0.0001$ & $<0.0001$ \\
Lack of fit & & 10 & 10 & & & & \\
R $^{2}$ & & 0.8758 & 0.9181 & & & & \\
Adj R & & 0.8427 & 0.8962 & & & & \\
Pred R & & 0.7781 & 0.8353 & & & & \\
C.V\% & 10.66 & 7.95 & & & & \\
\hline
\end{tabular}

A: extraction time $(1 \mathrm{~h}, 3 \mathrm{~h}, 5 \mathrm{~h})$; B: operating temperature $\left(20^{\circ} \mathrm{C}, 35^{\circ} \mathrm{C}, 50^{\circ} \mathrm{C}\right)$; C: peel-to-solvent ratio $(0.2,0.6$, $1.0 \mathrm{w} / \mathrm{v})$ 
It was also revealed from the data that the solid-to-liquid ratio was the only significant parameter affecting these three variables at linear and quadratic terms. The values of adjusted $\mathrm{R}^{2}$ and predicted $\mathrm{R}^{2}$ differ by less than 0.2 , meaning that these two values are in reasonable agreement with one another. The coefficients of variation, which measure the residual variability in the data, are expressed as percentage. Twenty treatments (runs) were conducted according to a central composite experimental design (Table 2). As the results of the different variables, the yield of colour compounds vary from $122.3 \mathrm{mg} \mathrm{l}^{-1}$ to $952.5 \mathrm{mg} \mathrm{l}^{-1}$ for betaxanthin and $148.5 \mathrm{mg} \mathrm{l}^{-1}$ to $1361 \mathrm{mg} \mathrm{l}^{-1}$ for betacyanin, respectively.

Table 2. Treatment variables and their corresponding responses

\begin{tabular}{|c|c|c|c|c|c|}
\hline Sample & Time (h) & Temperature $\left({ }^{\circ} \mathrm{C}\right)$ & $\begin{array}{c}\text { Solvent ratio } \\
(\mathrm{w} / \mathrm{v})\end{array}$ & $\begin{array}{l}\text { Betaxanthin } \\
\left(\mathrm{mg} \mathrm{l}^{-1}\right)\end{array}$ & $\begin{array}{c}\text { Betacyanin } \\
\left(\mathrm{mg} \mathrm{l}^{-1}\right)\end{array}$ \\
\hline 1 & 5 & 20 & 1 & 607.6 & 971.7 \\
\hline 2 & 3 & 20 & 0.6 & 670.1 & 1360.8 \\
\hline 3 & 3 & 35 & 0.6 & 829.2 & 1321.4 \\
\hline 4 & 1 & 20 & 1 & 952.5 & 1266.8 \\
\hline 5 & 1 & 35 & 0.6 & 662.0 & 1322.3 \\
\hline 6 & 5 & 20 & 0.2 & 191.9 & 472.5 \\
\hline 7 & 3 & 35 & 0.6 & 531.6 & 1031.7 \\
\hline 8 & 3 & 35 & 0.2 & 209.6 & 441.8 \\
\hline 9 & 3 & 35 & 1 & 592.7 & 1260.4 \\
\hline 10 & 1 & 50 & 0.2 & 170.0 & 347.9 \\
\hline 11 & 3 & 35 & 0.6 & 644.3 & 1122.0 \\
\hline 12 & 3 & 35 & 0.6 & 522.7 & 996.9 \\
\hline 13 & 3 & 35 & 0.6 & 753.2 & 1196.3 \\
\hline 14 & 3 & 35 & 0.6 & 556.7 & 1059.2 \\
\hline 15 & 5 & 50 & 0.2 & 122.3 & 148.5 \\
\hline 16 & 5 & 35 & 0.6 & 691.0 & 1200.8 \\
\hline 17 & 3 & 50 & 0.6 & 877.6 & 1273.3 \\
\hline 18 & 5 & 50 & 1 & 470.1 & 784.7 \\
\hline 19 & 1 & 50 & 1 & 564.1 & 1118.3 \\
\hline 20 & 1 & 20 & 0.2 & 167.2 & 331.8 \\
\hline
\end{tabular}

Even though the effects of extraction temperature and time on the colour compound yield were non-significant, lower extraction temperature and time were preferred to give better yield. The possible explanation may be the sensitivity of natural colour compounds to heat. Many phytochemicals are destroyed by high operation time and temperature as reported by HERBACH and co-workers (2006); AGUIRRE-JoYA and co-workers (2013); RAVICHANDRAN and co-workers (2013). Figure 1A and B shows the interaction effects of temperature and time on colour compounds when solvent ratio is maintained at constant level. 

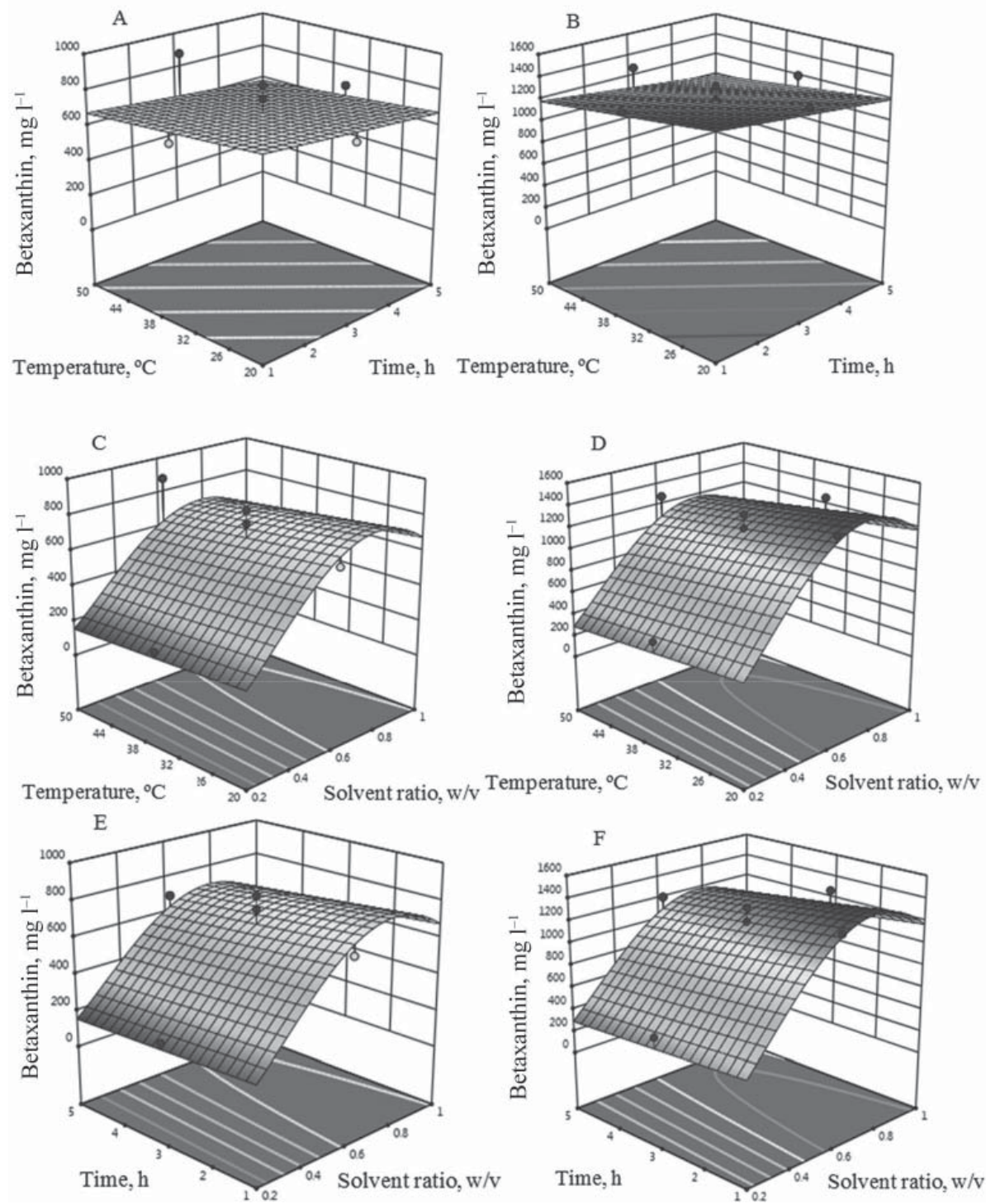

Fig. $1 A, B, C, D, E, F$. RSM for extractions of betaxanthin and betacyanin A: Correlational effect of temperature and time on betaxanthin quantity B: Correlational effect of temperature and time on betacyanin quantity C: Correlational effect of temperature and solvent ratio on betaxanthin quantity D: Correlational effect of temperature and solvent ratio on betacyanin quantity E: Correlational effect of time and solvent ratio on betaxanthin quantity F: Correlational effect of time and solvent ratio on betacyanin quantity

As it can be seen in Figure 1A and B, the decrease in time and temperature leads to increase in yield. This correlation can be explained by the findings of HERBACH and coworkers (2006), who investigated the impact of heating period on colour compounds. 
The significance of the quadratic term for the peel-to-solvent ratio indicates that this variable has impact on the extraction efficiency. According to Figure 1A, the highest yield of betaxanthin cannot be seen within the investigated time $(1-5 \mathrm{~h})$ and temperature $\left(20-50{ }^{\circ} \mathrm{C}\right)$ ranges, when the solvent ratio is fixed at centre point. It means that the yield mainly depends on the solvent ratio, which is in agreement with the findings of DE AzEREDO and co-workers (2009). On the contrary, in the case of betacyanin, the highest yield can be seen with the fixed solvent ratio in Figure 1B. It might be because of the different properties of specific colour compounds. The interaction effects of temperature and solvent ratio are depicted in Figure 1C and D, when time was fixed as constant. It was found that the yield increased when solvent ratio increased, with highest yield between $0.6 \mathrm{w} / \mathrm{v}$ and $1.0 \mathrm{w} / \mathrm{v}$ solvent ratio with low temperature around $20^{\circ} \mathrm{C}$ for betaxanthin compound. In case of betacyanin, the highest yield was obtained around $0.8 \mathrm{w} / \mathrm{v}$ of solvent ratio. Conversely, there was a minimal decrease in yield beyond that solvent ratio range for all colour compounds. The possible explanation is that the maximum extraction limit of colour compounds was reached, and further increment in solvent ratio became unnecessary for any driving force to occur with mass transfer. The same situation was observed when the temperature was fixed at $35^{\circ} \mathrm{C}$, providing the highest yield after $60 \mathrm{~min}$ as shown in Figure 1E and $\mathrm{F}$. The operating conditions were optimised to achieve the highest yields of basic colour compounds: betaxanthin and betacyanin with a desirability of 0.951 (Figure 2).
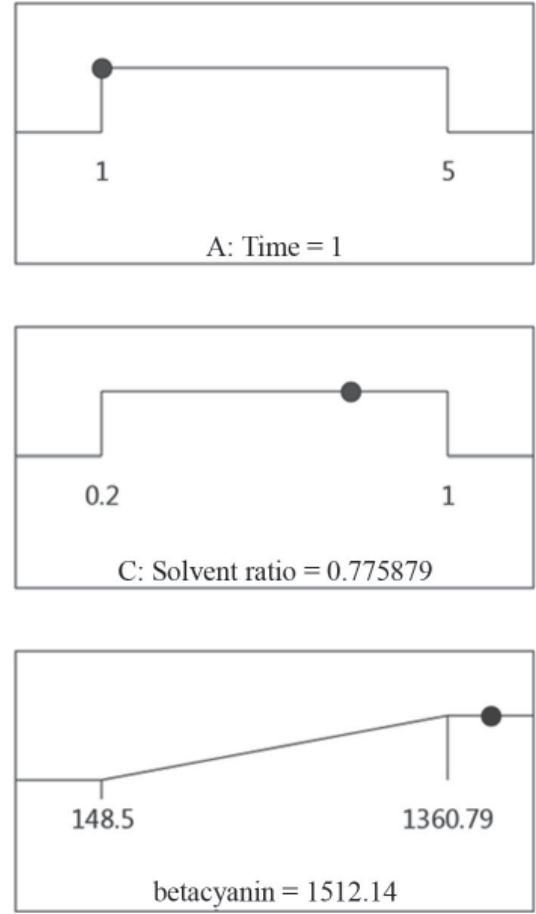
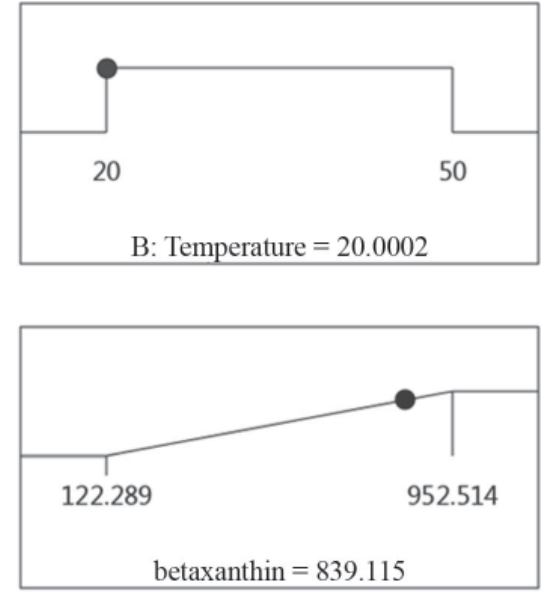

Desirability $=0.951$

Solution 1 out of 42

Fig. 2. Optimum condition for extraction of colour compounds with desirability of 0.951

The regression equations for obtaining betalain concentrations at any given parameters were given as

Sqrt (Betaxanthin) $\left(\mathrm{mg} \mathrm{l}^{-1}\right)=+5.00-0.44 \mathrm{~A}-0.06 \mathrm{~B}+65.99 \mathrm{C}-42.51 \mathrm{C}^{2}$

Sqrt (Betacyanin) $\left(\mathrm{mg} \mathrm{l}^{-1}\right)=+9.50-0.73 \mathrm{~A}-0.10 \mathrm{~B}+84.52 \mathrm{C}-55.46 \mathrm{C}^{2}$

Acta Alimentaria 49, 2020 


\section{Conclusions}

Central composite design used in this study suggested that the quadratic model with optimum conditions of extraction time $1 \mathrm{~h}$, operating temperature $20^{\circ} \mathrm{C}$, and solvent ratio $0.8 \mathrm{w} / \mathrm{v}$ gave a yield of $839.115 \mathrm{mg} \mathrm{l}^{-1}$ betaxanthin and $1512.14 \mathrm{mg} \mathrm{l}^{-1}$ betacyanin. Since we only used traditional extraction, our study encourages the use of this reasonably efficient method with cost efficiency.

The project is supported by the European Union and co-financed by the European Social Fund (grant agreement no. EFOP-3.6.3-VEKOP-16-2017-00005) and the Tempus Public Foundation under the Stipendium Hungaricum Scholarship Program.

\section{References}

Aguirre-Joya, J., De La Garza-Toledo, H., Zugasti-Cruz, A., Belmares-Cerda, R. \& Aguilar, C.N. (2013): The optimization of phenolic compounds extraction from cactus pear (Opuntia ficus-indica) skin in a reflux system using response surface methodology. Asian Pac. J. Trop. Biomed., 3(6), 436-442.

CAI, Y.Z., Sun, M. \& CoRke, H. (2005a): Characterization and application of betalain pigments from plants of the Amaranthaceae. Trends Food Sci. Tech., 16, 370-376.

CAI, Y.Z., Sun, M. \& CoRke, H. (2005b): HPLC Characterization of betalains from plants in the Amaranthaceae. J. Chromatogr. Sci., 43, 454-460.

De Azeredo, H.M.C., Pereira, A.C., De Souza, A.C.R., Gouveia, S.T. \& Mendes, K.C.B. (2009): Study on efficiency of betacyanin extraction from red beetroots. Int. J. Food Sci. Tech., 44, 2464-2469.

Georgiev, V.G., Weber, J., Kneschke, E.M., Denev, P.N., Bley, T. \& Pavlov, A.I. (2010): Antioxidant activity and phenolic content of betalain extracts from intact plants and hairy root cultures of the red beetroot Beta vulgaris cv. Detroit dark red. Plant Food Hum. Nutr., 65, 105-111.

Herbach, K.M., Stintzing, F.C. \& Carle, R. (2006): Stability and color changes of thermally treated betanin, phyllocactin, and hylocerenin solutions. J. Agr. Food Chem., 54, 390-398.

Kushwaha, R., Kumar, V., Vyas, G. \& KaUR, J. (2018): Optimization of different variable for eco-friendly extraction of betalains and phytochemicals from beetroot pomace. Waste Biomass Valori., 9, 1485-1494.

López, N., Puértolas, E., Condón, S., Raso, J. \& Alvarez, I. (2009): Enhancement of the extraction of betanine from red beetroot by pulsed electric fields. J. Food Eng., 90, 60-66.

Maran, J.P. \& PrIYA, B. (2016): Multivariate statistical analysis and optimization of ultrasound-assisted extraction of natural pigments from waste red beet stalks. J. Food Sci. Tech., 53(1), 792-799.

Ravichandran, K., Saw, N.M.T., Mohdaly, A.A.A., Gabr, A.M.M., ... \& Smetanska, I. (2013): Impact of processing of red beet on betalain content and antioxidant activity. Food Res. Int., 50(2), 670-675.

Singh, A., Ganesapillai, M. \& Gnanasundaram, N. (2017): Optimizaton of extraction of betalain pigments from beta vulgaris peels by microwave pretreatment. IOP Conf. Ser.: Mater. Sci. Eng., 263, 032004.

Sivakumar, V., AnNa, J.L., Vijayeeswarri, J. \& Swaminathan, G. (2009): Ultrasound assisted enhancement in natural dye extraction from beetroot for industrial applications and natural dyeing of leather. Ultrason. Sonochem., 16, 782-789.

Slimen, I.B., Najar, T. \& Abderrabba, M. (2017): Chemical and antioxidant properties of betalains. J. Agr. Food Chem. 65, 675-689.

Stintzing, F.C. \& CARLe, R. (2004): Functional properties of anthocyanins and betalains in plants, food, and in human nutrition. Trends Food Sci. Tech., 15, 19-38.

Open Access statement. This is an open-access article distributed under the terms of the Creative Commons Attribution 4.0 International License (https://creativecommons.org/licenses/by/4.0/), which permits unrestricted use, distribution, and reproduction in any medium, provided the original author and source are credited, a link to the CC License is provided, and changes - if any - are indicated. (SID_1) 